This item was submitted to Loughborough's Research Repository by the author.

Items in Figshare are protected by copyright, with all rights reserved, unless otherwise indicated.

\title{
Shared-control for the kinematic model of a mobile robot
}

PLEASE CITE THE PUBLISHED VERSION

https://doi.org/10.1109/cdc.2014.7039360

PUBLISHER

(c) IEEE

VERSION

AM (Accepted Manuscript)

LICENCE

CC BY-NC-ND 4.0

REPOSITORY RECORD

Jiang, Jingjing, and Alessandro Astolfi. 2019. "Shared-control for the Kinematic Model of a Mobile Robot". figshare. https://hdl.handle.net/2134/36759. 


\title{
Shared-Control for the Kinematic Model of a Mobile Robot
}

\author{
Jingjing Jiang $^{1}$ and Alessandro Astolfi ${ }^{2}$
}

\begin{abstract}
This paper presents a shared-control algorithm for the kinematic model of a mobile robot. The set of feasible position of the robot is defined by a group of linear inequalities. The shared-control strategy is based on a hysteresis switch and its properties are established by a Lyapunov-like analysis. Simulation results illustrate the effectiveness of the algorithm.
\end{abstract}

\section{INTRODUCTION}

This paper deals with the shared-control problem for a simple mobile robot. The robot is "driven" by a human operator which is "supervised" by a feedback controller. The human operator provides velocity commands the robot except for "emergency" situations, in which the feedback controller is active.

Mobile robots are widely used in probing [1], cleaning [2], military applications [3], industries [4], [5] and household tasks [6]. The present paper proposes a shared-control method to drive a wheeled mobile robot in the case in which the control authority is shared by a human operator and a feedback controller. This shared-control system is widely used in the modern world, for example in intelligent wheelchairs [7], teleoperations [8], transportations [9] and medicine [10]. The paper [11] has shown that there are four types of robot modes according to the level of system's autonomy and has demonstrated that collaborative control can increase performance and reduce error compared with manual operation, whereas [12] has introduced the idea of task-level adaptive shared-control to assembly systems and demonstrated that the resulting system is flexible and able to accomodate changes. The control authority shared between a human operator and a feedback controller is similar to that shared between two operators, which is commonly used in training systems [13]. For example, the paper [14] has quantified how much the interaction between two users and a slave robot, as well as the environment, occurs in a dualuser teleoperation system through a dominance factor which is chosen by means of experimental trials.

Shared control problems have been studied in [15], where a continuous shared control paradigm based on a constant sharing weight determined via simulations has been introduced for a brain-machine interface commanding a robot in

\footnotetext{
${ }^{1} \mathrm{~J}$. Jiang is with the Department of Electrical and Electronic Engineering, Imperial College London, UK, E-mail: jingjing.jiang10@imperial.ac.uk

${ }^{2} \mathrm{~A}$. Astolfi is with the Dept. of Electrical and Electronic Engineering, Imperial College London, London, SW7 2AZ, UK and the DICII, University of Roma "Tor Vergata", Via del Politecnico 1, 00133 Rome, Italy, E-mail: a.astolfieimperial.ac.uk
}

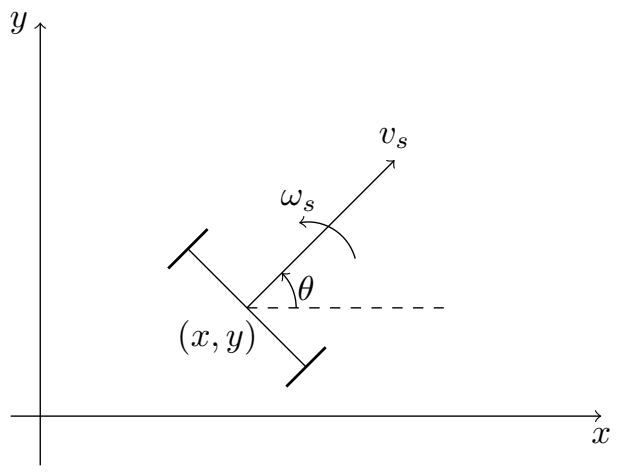

Fig. 1. Kinematic Model for a Unicycle-like Mobile Robot

reach and grasp tasks. In [16] the sharing weight is a variable calculated on-line. Finally in [17] and [18] the shared-control problem for fully actuated, linear, mechanical systems has been studied by utilizing ideas from [19] and [20].

In shared-control the aim is to let the human operator in charge of the system in "normal" conditions, while the feedback control works only in "dangerous" situations: this is similar to an obstacle avoidance problem. The obstacles are characterized by regions that cannot be visited by the robot depending on the states and the input. The potential field method is a commonly used way to solve the collision avoidance problem, but suffers from significant shortcomings discussed for example in [21], such as the existence of local minimizers and oscillatory trajectories when passing through a narrow corridor. These drawbacks can be partly alleviated with the use of Lyapunov-like methods [22]. However, this approach prevents the configuration of the system from reaching the boundary of the obstacle. This limitation can be overcome by the shared-control law presented in this paper, which allows the robot to move safely within a predefined, boundary-reachable, admissible set.

The rest of the paper is organized as follows. The problem is formulated in Section II together with some assumptions. The design of the shared-control is given in Section III where formal properties of the closed-loop system are presented. In Section IV two numerical examples to illustrate the performance of the shared-control are given. Finally, Section V gives some conclusions and suggestions for future work.

\section{PROBLEM STATEMENT}

In this section we formulate the shared-control problem for the kinematic model of a mobile robot. For simplicity we 
assume that the robot is a wheeled robot (see Figure 1), the dynamics of which are described by the equations

$$
\begin{aligned}
\dot{x} & =v_{s} \cos \theta, \\
\dot{y} & =v_{s} \sin \theta, \\
\dot{\theta} & =\omega_{s},
\end{aligned}
$$

where $(x, y)$ denotes the Cartesian coordinates of the center of the robot's mass, $\theta$ represents the angle between the (positive) heading direction and the $x$-axis, $v_{s}$ and $\omega_{s}$ are the linear velocity and the angular velocity of the mobile robot, respectively. Note that $v_{s}$ and $\omega_{s}$ are the inputs of the system.

Let

$$
\begin{aligned}
v_{s}(x, y, \theta, t)= & {\left[1-k\left(x, y, \theta, v_{h}\right)\right] v_{f}(x, y, \theta, t) } \\
& +k\left(x, y, \theta, v_{h}\right) v_{h}(x, y, \theta, t), \\
\omega_{s}(x, y, \theta, t)= & {\left[1-k\left(x, y, \theta, v_{h}\right)\right] \omega_{f}(x, y, \theta, t) } \\
& +k\left(x, y, \theta, v_{h}\right) \omega_{h}(x, y, \theta, t),
\end{aligned}
$$

where $u_{h}=\left[v_{h}, \omega_{h}\right]^{T}$, denoted as $h$-control, describes the human action, $u_{f}=\left[v_{f}, \omega_{f}\right]^{T}$, denoted as $f$-control, represents the feedback-control action and $k$, denoted as sharing function, quantifies how the control action is shared. In what follows we use the name s-closed-loop to denote the system described by equation (1) with input $u_{s}=\left[v_{s}, \omega_{s}\right]^{T}$ given by equation (2) and the name $h$-closed-loop to denote the system described by the equations

$$
\begin{aligned}
\dot{x} & =v_{h} \cos \theta, \\
\dot{y} & =v_{h} \sin \theta, \\
\dot{\theta} & =\omega_{h} .
\end{aligned}
$$

Note that the s-closed-loop system and the h-closed-loop system share the same state space $\mathcal{P} \times \mathcal{A}=\mathbb{R}^{2} \times \mathbb{S}$, where $\mathcal{P}$ denotes the set of Cartesian positions in the plane and $\mathcal{A}$ denotes the set of heading angles. Suppose $\mathcal{P}_{a} \in \mathcal{P}$ is a given, closed and compact set that describes the admissible Cartesian configuration set for the system (1) and $u_{h}$ is a given h-control. The shared-control problem can be posed as follows.

Given the system (1), an admissible configuration set $\mathcal{P}_{a}$ and an h-control $u_{h}$, find (if possible)

- an f-control $u_{f}$;

- a sharing function $k$;

- a safe set $\mathcal{R}_{s}\left(v_{h}\right) \triangleq \mathcal{P}_{a} \times \mathcal{A}_{s} \subset \mathcal{P}_{a} \times \mathcal{A} \triangleq \mathcal{R}\left(v_{h}\right)$;

such that the s-closed-loop system (1)-(2) has the following properties.

P1) The set $\mathcal{R}\left(v_{h}\right)$ is forward invariant.

P2) Let $\Omega_{s}$ and $\Omega_{h}$ be the $\Omega$-limit set of the s-closed-loop and h-closed-loop, respectively. Then

$$
\Omega_{s}= \begin{cases}\Omega_{h} & \text { if } \Omega_{h} \subset \mathcal{R}_{s}\left(v_{h}\right), \\ \Pi_{\mathcal{R}_{s}}\left(\Omega_{h}\right) & \text { if } \Omega_{h} \not \subset \mathcal{R}_{s}\left(v_{h}\right),\end{cases}
$$

where $\Pi_{\mathcal{R}_{s}}\left(\Omega_{h}\right)$ is the projection of $\Omega_{h}$ into the set $\mathcal{R}_{s}\left(v_{h}\right)$, which will be defined in Section III-A.

P3) $u_{s}=u_{h}$ if $(x, y, \theta) \in \mathcal{R}_{s}\left(v_{h}\right)$.

Note that for any fixed $v_{h}$ and any $p \in \mathcal{P}_{a}, \mathcal{A}_{s}$ is the set of all possible heading angles with which the robot is unable to hit the boundary of $\mathcal{P}_{a}$ within a short time.

\section{DESIGN OF THE SHARED-CONTROL}

In this section we give a solution to the shared-control problem presented in Section II in the case in which $\mathcal{P}_{a}$ is described by a group of linear inequalities, namely

$$
\mathcal{P}_{a}=\left\{p \in \mathbb{R}^{2} \mid S p+T \leq 0\right\},
$$

where $p=[x, y]^{T}, S=\left[s_{1}^{T}, s_{2}^{T}, \ldots, s_{m}^{T}\right]^{T} \in \mathbb{R}^{m \times 2}$ and $T=\left[t_{1}, t_{2}, \ldots, t_{m}\right]^{T} \in \mathbb{R}^{m}$. Similarly to [17] and [18] we make the following assumption throughout the paper.

Assumption 1: If $m>2$ then the matrices $S$ and $T$ are such that

$$
\operatorname{rank}\left(\left[\begin{array}{l}
s_{r_{1}} \\
\vdots \\
s_{r_{l}}
\end{array}\right]\right)<\operatorname{rank}\left(\left[\begin{array}{ll}
s_{r_{1}} & t_{r_{1}} \\
\vdots & \vdots \\
s_{r_{l}} & t_{r_{l}}
\end{array}\right]\right),
$$

for all $l \in[3, m]$ and $r_{1}, r_{2}, \ldots, r_{l} \in\{1,2, \ldots, m\}$.

\section{A. Design of the f-control}

In this section we design the f-control under the assumption $m=2$. As discussed in [17] this is without loss of generality. In addition, as noted therein, $N_{c}$ f-controls need to be designed and in general $N_{c} \leq\left(\begin{array}{c}m \\ 2\end{array}\right)$. Consider now the $i^{\text {th }}$ group of constraints

$$
S^{i} p+T^{i} \leq 0
$$

where $S^{i}=\left[s_{1}^{i^{T}}, s_{2}^{i}\right]^{T} \in \mathbb{R}^{2 \times 2}$ and $T=\left[t_{1}^{i}, t_{2}^{i}\right]^{T} \in \mathbb{R}^{2}$. Note that, as detailed in [17], $S^{i}$ is invertible according to its construction. Define new variables $q^{i}$ and $z^{i}=\left[z_{1}^{i}, z_{2}^{i}\right]^{T}$ as

$$
q^{i}=S^{i} p+T^{i}
$$

and

$$
z_{j}^{i}=\log \frac{q_{j}^{i}}{q_{r_{j}}^{i}},
$$

for all $j \in\{1,2\}$, where $q_{r_{j}}^{i}$ (to be defined) describes the reference trajectory for the state $q_{j}^{i}$.

Define $q_{r}^{i}=\left[q_{r_{1}}^{i}, q_{r_{2}}^{i}\right]^{T}$ as

$$
q_{r_{j}}^{i}= \begin{cases}q_{d}^{i}, & \text { if } q_{d_{j}}^{i} \leq\left(1-\frac{\sqrt{2}}{2}\right) r-\epsilon, \\ -\epsilon, & \text { if } q_{d_{j}}^{i} \geq(\sqrt{2}-1) r-\epsilon, \\ h_{j}^{i}, & \text { otherwise },\end{cases}
$$

for $j \in\{1,2\}$, where $r$ is a positive constant, $h_{j}^{i}=-(r+\epsilon)+$ $\sqrt{r^{2}-\left[(\sqrt{2}-1) r-\epsilon-q_{d_{j}}^{i}\right]^{2}}, q_{d}^{i}=\left[q_{d_{1}}^{i}, q_{d_{2}}^{i}\right]^{T}=S^{i} p_{d}+$ 
$T^{i}$ and $p_{d}$ refers to the reference signal in the space $\mathcal{P}$. Note that, by definition, $q_{r_{j}}^{i}$ is a smooth and non-positive function. As a result, $\dot{q}_{r_{j}^{i}}$ and $\ddot{q}_{r_{j}^{i}}$ exist. Finally, define $\left(p_{r}^{i}, \alpha_{r}^{i}, v_{r}^{i}, \theta_{r}^{i}\right)$ as

$$
\begin{aligned}
p_{r}^{i} & =S^{i^{-1}}\left(q_{r}^{i}-T^{i}\right), \\
\alpha_{r}^{i} & =S^{i^{-1}} \dot{q}_{r}^{i}, \\
v_{r}^{i} & =\sqrt{\alpha_{r_{1}}^{i}+\alpha_{r_{2}}^{i}}, \\
\theta_{r}^{i} & =\operatorname{atan} 2\left(\alpha_{r_{2}}^{i}, \alpha_{r_{1}}^{i}\right) .
\end{aligned}
$$

Suppose $\left(p_{d}, \theta_{d}\right)$ is a point of the $\Omega$-limit set of the h-closedloop, i.e. $\left(p_{d}, \theta_{d}\right) \in \Omega_{h}$, and define the projection of $\left(p_{d}, \theta_{d}\right)$ into $\mathcal{R}_{s}$, relative to the $i^{\text {th }}$ group of constraints, by

$$
\Pi_{\mathcal{R}_{s}}^{i}\left(p_{d}, \theta_{d}\right)=\left(p_{r}^{i}, \theta_{r}^{i}\right)
$$

where $\left(p_{r}^{i}, \theta_{r}^{i}\right)$ is defined by (7). Then the projection of $\Omega_{h}$ into the safe set $\mathcal{R}_{s}$ is defined by

$$
\Pi_{\mathcal{R}_{s}}^{i}\left(\Omega_{h}\right)=\left\{s \in \mathcal{R}_{s} \mid s=\Pi_{\mathcal{R}_{s}}^{i}\left(p_{d}, \theta_{d}\right)\right\}, \forall\left(p_{d}, \theta_{d}\right) \in \Omega_{h}
$$

In addition, for any given $\alpha_{r}^{i}$, the reference input signal $u_{r}^{i}$ related to the $i^{\text {th }}$ group of active constraints is defined as

$$
u_{r}^{i}=\left[\begin{array}{c}
v_{r}^{i} \\
\omega_{r}^{i}
\end{array}\right]=\left[\begin{array}{c}
\sqrt{\alpha_{r_{1}}^{i^{2}}+\alpha_{r_{2}}^{{ }^{2}}} \\
\frac{\mathrm{d}}{\mathrm{dt}}\left(\operatorname{atan} 2\left(\alpha_{r_{1}}^{i}, \alpha_{r_{2}}^{i}\right)\right)
\end{array}\right] .
$$

With the new variable $z^{i}$ and the feedback controller $u_{f}^{i}$, the $i^{\text {th }}$ group of constraints on $p$, i.e. $S^{i} p+T^{i} \leq 0$, can be removed and the system (1) can be rewritten as

$$
\begin{aligned}
& \dot{z}_{1}^{i}=\frac{v_{f}^{i} \cos \theta^{i}}{e^{z_{1}^{i}} q_{r_{1}}^{i}}-\frac{v_{r}^{i} \cos \theta_{r}^{i}}{q_{r_{1}}^{i}} \\
& \dot{z}_{2}^{i}=\frac{v_{f}^{i} \cos \theta^{i}}{e^{z_{2}^{i}} q_{r_{2}}^{i}}-\frac{v_{r}^{i} \cos \theta_{r}^{i}}{q_{r_{2}}^{i}} \\
& \dot{\theta}^{i}=\omega_{f}^{i} .
\end{aligned}
$$

Let

$$
\theta^{i *}=\operatorname{atan} 2\left(e^{z_{2}^{i}}\left(\alpha_{r}^{i} \sin \theta_{r}^{i}-\gamma_{2} z_{2}^{i}\right), e^{z_{1}^{i}}\left(\alpha_{r}^{i} \cos \theta_{r}^{i}-\gamma_{1} z_{1}^{i}\right)\right)
$$

where $\gamma_{1}>0$ and $\gamma_{2}>0$.

Consider now the $i^{\text {th }}$ Lyapunov function $L^{i}\left(z_{1}^{i}, z_{2}^{i}, \theta^{i}\right)$ given by the equation

$$
L^{i}\left(z_{1}^{i}, z_{2}^{i}, \theta^{i}\right)=\frac{1}{2}\left[z_{1}^{i^{2}}+z_{2}^{i^{2}}+\left(\theta^{i}-\theta^{i *}\right)^{2}\right]
$$

and choose $v_{f}^{i}$ and $\omega_{f}^{i}$ such that $\dot{L}^{i}<0$ for all $\left(z_{1}^{i}, z_{2}^{i}\right) \neq$
$(0,0)$ and $\theta^{i} \neq \theta^{i *}$. One such a choice is given by

$$
\begin{aligned}
& v_{f}^{i}=\sqrt{\begin{array}{c}
e^{2 z_{1}^{i}}\left(v_{r}^{i} \cos \theta_{r}^{i}-\gamma_{1} z_{1}^{i}\right)^{2} \\
+e^{2 z_{2}^{i}}\left(v_{r}^{i} \sin \theta_{r}^{i}-\gamma_{2} z_{2}^{i}\right)^{2}
\end{array}}, \\
& \omega_{f}^{i}=\dot{\theta}^{i *}-\gamma_{3}\left(\theta^{i}-\theta^{i *}\right)-\frac{z_{2}^{i} v_{f}^{i} \cos \frac{\theta^{i}+\theta^{i *}}{2} \operatorname{sinc} \frac{\theta^{i}-\theta^{i *}}{2}}{q_{2}^{i}} \\
& +\frac{z_{1}^{i} v_{f}^{i} \sin \frac{\theta^{i}+\theta^{i *}}{2} \operatorname{sinc} \frac{\theta^{i}-\theta^{i *}}{2}}{q_{1}^{i}} .
\end{aligned}
$$

This control can be pull back to the $\left(q^{i}, \theta\right)$ coordinates giving

$$
\begin{gathered}
v_{f}^{i}=\sqrt[\begin{array}{c}
\left(\frac{q_{1}^{i}}{q_{r_{1}}^{i}}\right)^{2}\left(v_{r}^{i} \cos \theta_{r}^{i}-\gamma_{1} \log \frac{q_{1}^{i}}{q_{r_{1}}^{i}}\right)^{2} \\
+\left(\frac{q_{2}^{i}}{q_{r_{2}}^{i}}\right)^{2}\left(v_{r}^{i} \sin \theta_{r}^{i}-\gamma_{2} \log \frac{q_{2}^{i}}{q_{r_{2}}^{i}}\right)^{2}
\end{array}]{\omega_{f}^{i}=\frac{\log \frac{q_{1}^{i}}{q_{r_{1}}^{i}} v_{f}^{i} \sin \frac{\theta^{i}+\theta^{i *}}{2} \operatorname{sinc} \frac{\theta^{i}-\theta^{i *}}{2}}{q_{1}^{i}}} \\
+\frac{\log \frac{q_{2}^{i}}{q_{r_{2}}^{i}} v_{f}^{i} \cos \frac{\theta^{i}+\theta^{i *}}{2} \operatorname{sinc} \frac{\theta^{i}-\theta^{i *}}{2}}{q_{2}^{i}} \\
+\dot{\theta}^{i *}-\gamma_{3}\left(\theta^{i}-\theta^{i *}\right),
\end{gathered}
$$

where

$$
\theta^{i *}=\operatorname{atan} 2\left(\begin{array}{c}
\frac{q_{2}^{i}}{q_{r_{2}}^{i}}\left(\alpha_{r_{2}}^{i} \sin \theta_{r}^{i}-\gamma_{2} \log \frac{q_{2}^{i}}{q_{r_{2}}^{i}}\right), \\
\frac{q_{1}^{i}}{q_{r_{1}}^{i}}\left(\alpha_{r_{1}}^{i} \cos \theta_{r}^{i}-\gamma_{1} \log \frac{q_{1}^{i}}{q_{r_{1}}^{i}}\right)
\end{array}\right)
$$

and $q_{j}^{i}=s_{j}^{i} p+t_{j}^{i}, q_{r_{j}}^{i}=s_{j}^{i} p_{r}+t_{j}^{i}$ for all $j \in\{1,2\}$.

Lemma 1: Consider the f-closed-loop system (1)-(9) with $\left[v_{s}, \omega_{s}\right]^{T}=\left[v_{f}^{i}, \omega_{f}^{i}\right]^{T}$ given by (11), $q_{r}^{i}$ given by (6), and $v_{r}^{i}$ and $\theta_{r}^{i}$ given by (7). Assume $(x(0), y(0)) \in \mathcal{P}_{a}$. Then the system has the following properties.

P1) $(x(t), y(t)) \in \mathcal{P}_{a}$ for all $t \geq 0$;

P2) $\lim _{t \rightarrow \infty}\left(x(t)-p_{r_{1}}(t)\right)=\lim _{t \rightarrow \infty}\left(y(t)-p_{r_{2}}(t)\right)=0$.

\section{B. Shared Control Theorem}

By Property (P3) in Section II we need to find the safe subset $\mathcal{R}_{s}$ before designing the sharing function $k$. Relative to the $i^{\text {th }}$ group of constraints and a given h-control $v_{h}$, the safe, hysteresis and dangerous subsets $\tilde{\mathcal{R}}_{s}^{i}, \tilde{\mathcal{R}}_{h}^{i}$ and $\tilde{\mathcal{R}}_{d}^{i}$ are defined in equations (12) on the top of the next page, where ${ }^{1} \mathcal{Q}_{a}^{i}=$

\footnotetext{
${ }^{1}$ The notation $S \mathcal{P}+T$, with $S \in \mathbb{R}^{2 \times 2}, T \in \mathbb{R}^{2}$, and $\mathcal{P} \in \mathbb{R}^{2}$ denotes the set defined as

$$
\left\{x \in \mathbb{R}^{2} \mid x=S y+T, y \in \mathcal{P}\right\} .
$$
}




$$
\begin{aligned}
& \tilde{\mathcal{R}}_{s}^{i}\left(v_{h}\right)=\left\{\left(q^{i}, \theta^{i}\right) \in \mathcal{Q}_{a}^{i} \times \mathbb{S}:\left(s_{j}^{i}\left[\cos \theta^{i}, \sin \theta^{i}\right]^{T} v_{h}\right) \leq \frac{1}{q_{j}^{i}+b_{2}}-\frac{1}{b_{2}} \text { if } q_{j}^{i} \geq-b_{2} \text { for all } j \in\{1,2\}\right\} \\
& \tilde{\mathcal{R}}_{h}^{i}\left(v_{h}\right)=\left\{\begin{aligned}
\left(q^{i}, \theta^{i}\right) \in \mathcal{Q}_{a}^{i} \times \mathbb{S}: & \exists j \in\{1,2\} \text { such that }\left(s_{j}^{i}\left[\cos \theta^{i}, \sin \theta^{i}\right]^{T} v_{h}\right)>\frac{1}{q_{j}^{i}+b_{2}}-\frac{1}{b_{2}} \text { and } q_{j}^{i} \geq-b_{2} \\
& \text { and }\left(s_{k}^{i}\left[\cos \theta^{i}, \sin \theta^{i}\right]^{T} v_{h}\right)<\frac{1}{q_{k}^{i}+b_{1}}-\frac{1}{b_{1}} \text { if } q_{k}^{i} \geq-b_{1} \text { for all } k \in\{1,2\}
\end{aligned}\right\} \\
& \tilde{\mathcal{R}}_{d}^{i}\left(v_{h}\right)=\left\{\begin{array}{cc}
\left(q^{i}, \theta^{i}\right) \in \mathcal{Q}_{a}^{i} \times \mathbb{S}: & \exists j \in\{1,2\} \text { such that }\left(s_{j}^{i}\left[\cos \theta^{i}, \sin \theta^{i}\right]^{T} v_{h}\right) \geq \frac{1}{q_{j}^{i}+b_{1}}-\frac{1}{b_{1}} \text { and }-b_{1} \leq q_{j}^{i}<0 \\
& \text { or } \exists j \in\{1,2\} \text { such that }\left(s_{j}^{i}\left[\cos \theta^{i}, \sin \theta^{i}\right]^{T} v_{h}\right)>\frac{1}{q_{j}^{i}+b_{1}}-\frac{1}{b_{1}} \text { and } q_{j}^{i}=0 \\
& \text { or } \forall j \in\{1,2\} \text { such that } q_{j}^{i}=\left(s_{j}^{i}\left[\cos \theta^{i}, \sin \theta^{i}\right]^{T} v_{h}\right)=0
\end{array}\right\}
\end{aligned}
$$

$S^{i} \mathcal{P}_{a}+T^{i}$, and $b_{2}>b_{1}>0$. Note that $\tilde{\mathcal{R}}_{s}^{i}\left(v_{h}\right), \tilde{\mathcal{R}}_{h}^{i}\left(v_{h}\right)$ and $\tilde{\mathcal{R}}_{d}^{i}\left(v_{h}\right)$ are defined in the $\left(q^{i}, \theta^{i}\right)$ coordinates and can be pull back to the $(p, \theta)$ coordinates by the relations

$$
\begin{aligned}
& \mathcal{R}_{s}^{i}\left(v_{h}\right)=\operatorname{diag}\left(\mathrm{S}^{\mathrm{i}-1}, 1\right)\left(\tilde{\mathcal{R}}_{\mathrm{s}}^{\mathrm{i}}-\operatorname{col}\left(\mathrm{T}^{\mathrm{i}}, 0\right)\right), \\
& \mathcal{R}_{h}^{i}\left(v_{h}\right)=\operatorname{diag}\left(\mathrm{S}^{\mathrm{i}-1}, 1\right)\left(\tilde{\mathcal{R}}_{\mathrm{h}}^{\mathrm{i}}-\operatorname{col}\left(\mathrm{T}^{\mathrm{i}}, 0\right)\right), \\
& \mathcal{R}_{d}^{i}\left(v_{h}\right)=\operatorname{diag}\left(\mathrm{S}^{\mathrm{i}-1}, 1\right)\left(\tilde{\mathcal{R}}_{\mathrm{d}}^{\mathrm{i}}-\operatorname{col}\left(\mathrm{T}^{\mathrm{i}}, 0\right)\right),
\end{aligned}
$$

where $\operatorname{col}\left(\mathrm{T}^{\mathrm{i}}, 0\right)$ is a column vector obtained by stacking the number 0 under the vector $T^{i}$.

By construction, $\mathcal{R}_{s}^{i}\left(v_{h}\right), \mathcal{R}_{h}^{i}\left(v_{h}\right)$ and $\mathcal{R}_{d}^{i}\left(v_{h}\right)$ have the following properties:

- $\mathcal{R}_{s}^{i}\left(v_{h}\right) \cup \mathcal{R}_{h}^{i}\left(v_{h}\right) \cup \mathcal{R}_{d}^{i}\left(v_{h}\right)=\mathcal{R}\left(v_{h}\right)$ for all $i \in$ $\left\{1,2, \ldots, N_{c}\right\}$;

- $\mathcal{R}_{d}^{i}\left(v_{h}\right) \cap \mathcal{R}_{d}^{j}\left(v_{h}\right)=\emptyset$ for all $i \neq j$ and $i, j \in$ $\left\{1,2, \ldots, N_{c}\right\}$

- $\mathcal{R}_{s}^{i}\left(v_{h}\right) \cap \mathcal{R}_{d}^{i}\left(v_{h}\right)=\left\{\left(p, v_{h}\right) \mid S^{i} p+T^{i}=0, v_{h}=0\right\}$;

- $\mathcal{R}_{d}\left(v_{h}\right)=\mathcal{R}_{d}^{1}\left(v_{h}\right) \cup \cdots \cup \mathcal{R}_{d}^{N_{c}}\left(v_{h}\right)$,

$\mathcal{R}_{h}\left(v_{h}\right)=\mathcal{R}_{h}^{1}\left(v_{h}\right) \cup \cdots \cup \mathcal{R}_{h}^{N_{c}}\left(v_{h}\right)$,

$\mathcal{R}_{s}\left(v_{h}\right)=\mathcal{R}_{s}^{1}\left(v_{h}\right) \cap \cdots \cap \mathcal{R}_{s}^{N_{c}}\left(v_{h}\right)$.

For each group of constraints, the sharing function $k^{i}$ can be defined as, see [23],

$k^{i}\left(p, \theta, v_{h}\right)= \begin{cases}1, & (p, \theta) \in \mathcal{R}_{s}^{i}\left(v_{h}\right) \backslash \mathcal{R}_{d}^{i}\left(v_{h}\right), \\ l^{i}\left(p, \theta, v_{h}\right), & (p, \theta) \in \mathcal{R}_{h}^{i}\left(v_{h}\right), \\ 0, & (p, \theta) \in \mathcal{R}_{d}^{i}\left(v_{h}\right),\end{cases}$

where

$l^{i}\left(p, \theta, v_{h}\right)= \begin{cases}1, & \text { if }(p, \theta) \text { enters } \mathcal{R}_{h}^{i}\left(v_{h}\right) \text { from } \mathcal{R}_{s}^{i}\left(v_{h}\right), \\ 0, & \text { if }(p, \theta) \text { enters } \mathcal{R}_{h}^{i}\left(v_{h}\right) \text { from } \mathcal{R}_{d}^{i}\left(v_{h}\right) .\end{cases}$

Finally, the s-control is given by the equation

$$
\begin{aligned}
u_{s}\left(p, \theta, v_{h}\right)= & \sum_{i=1}^{N_{c}}\left[\left(1-k^{i}\left(p, \theta, v_{h}\right)\right) u_{f}^{i}\left(p, p_{r}, \theta_{r}, \alpha_{r}\right)\right] \\
& +\min _{i=1}^{N_{c}} k^{i}\left(p, \theta, v_{h}\right) u_{h} .
\end{aligned}
$$

Lemma 2: Consider the system (1) with the shared-control input (11), (13), (14). Let $(p(t), \theta(t))$ be a trajectory of the system. Assume $(p(0), \theta(0)) \in \mathcal{R}_{s}\left(v_{h}(0)\right)$. Suppose there exists $\bar{t}>0$ such that $(p(\bar{t}), \theta(\bar{t})) \notin \mathcal{R}\left(v_{h}(\bar{t})\right)$. Then there exists a $t_{d}$ such that $0<t_{d}<\bar{t}$ and $\left(p\left(t_{d}\right), \theta\left(t_{d}\right)\right) \in$ $\mathcal{R}_{d}\left(v_{h}\left(t_{d}\right)\right)$.

Theorem 1: Consider the kinematic model (1) of a mobile robot with a given h-control $u_{h}$ and the shared-control law given by (11)-(13)-(14). Assume the admissible configuration set $\mathcal{P}_{a}$ is defined by (3), $p(0) \in \mathcal{P}_{a}$ and the $\Omega$-limit set $\Omega_{h}$ of the h-closed-loop is safe, i.e. $\Omega_{h} \subset \mathcal{R}_{s}$. Then there exist $\gamma_{i}>0$, for all $i \in\{1,2,3\}$, and $b_{2}>b_{1}>0$ such that the s-closed-loop system has the following properties.

(1) $p(t)$ stays in $\mathcal{P}_{a}$ for all $t \geq 0$.

(2) $\Omega_{s}=\Omega_{h}$.

(3) For all $t \geq 0$ such that $(p(t), \theta(t)) \in \mathcal{R}_{s}\left(v_{h}(t)\right) \backslash$ $\mathcal{R}_{d}\left(v_{h}(t)\right), u_{s}(t)=u_{h}(t)$.

Remark 1: If $\Omega_{h} \not \subset \mathcal{R}_{s}$, then claim (2) in Theorem 1 should be modified as: $\Omega_{s}=\Pi_{\mathcal{R}_{s}}\left(\Omega_{h}\right)$.

\section{NUMERICAL EXAMPLES}

In this section we discuss two case studies: in the first case $\mathcal{P}_{a}$ is convex and in the second case $\mathcal{P}_{a}$ is non-convex. Note that the state of the h-closed-loop system goes outside of $\mathcal{P}_{a}$ in both cases, i.e. $\Omega_{h} \not \subset \mathcal{R}_{s}$.

\section{A. Convex $\mathcal{P}_{a}$}

Consider the kinematic model (1) and the admissible region $\mathcal{P}_{a}$ defined by

$$
\mathcal{P}_{a}=\{(x, y) \mid x \geq 2, y \geq 2\} .
$$

Let the human operator generate a random $(x, y)$ trajectory, the red, dashed-and-dotted, curve in Figure 2. The corresponding $(x, y)$ trajectory of the s-closed-loop system is displayed by the green, dashed, curve in Figure 2. Figure 3 shows how the inputs and states of the h-closed-loop and 
s-closed-loop systems vary with time. $v_{s}(t) \neq v_{h}(t)$ and $\omega_{s}(t) \neq \omega_{h}(t)$ when $t \in(25,28)$ and $t \in(34,41)$ implies that the $\mathrm{f}$-control is active and $k=0$. With the sharedcontrol, the robot moves along the boundary of $\mathcal{P}_{a}$ until the reference trajectory (i.e. the trajectory of the h-closed-loop) returns to the admissible set.

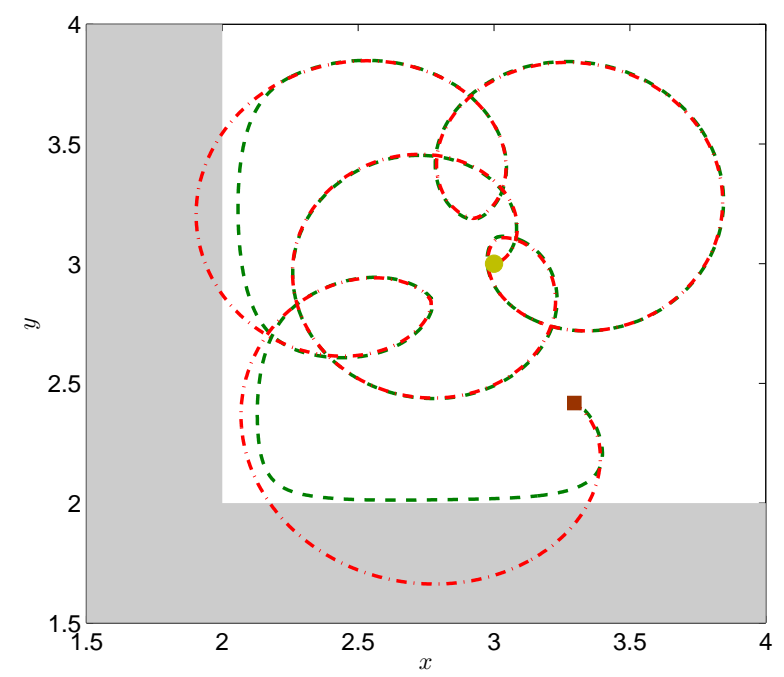

Fig. 2. $\quad(x, y)$ trajectories of the system (1) for the set $\mathcal{P}_{a}$ given in (15): h-closed-loop (red, dashed-and-dotted) and s-closed-loop (green, dashed). Round mark: the initial position of the robot. Square mark: the final position of the robot.
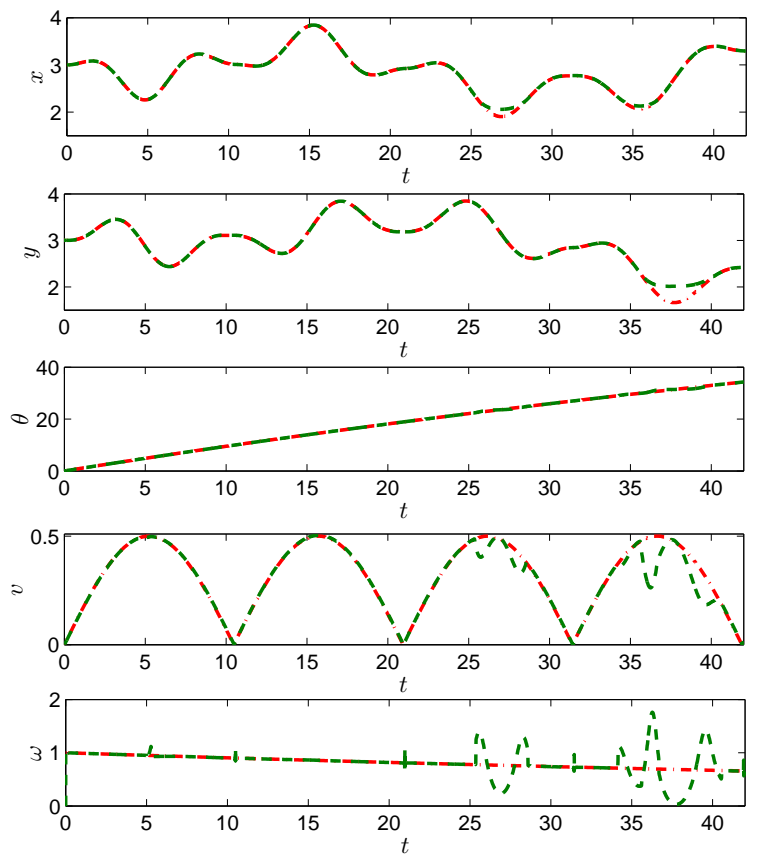

Fig. 3. Time histories of the variable $x, y, \theta, v$ and $\omega$ for the system (1) with the set $\mathcal{P}_{a}$ given by (15): h-closed-loop system (red, dashed-anddotted) and s-closed-loop system (green, dashed).

\section{B. Non-convex $\mathcal{P}_{a}$}

As stated in [18], the shared-control algorithm can also be applied to non-convex admissible configuration sets defined by a group of linear inequalities complemented with logic conditions. To illustrate this scenario consider the system (1) and the non-convex region

$$
\mathcal{P}_{a}=\left\{\begin{array}{l|c}
0 \leq x \leq 6,0 \leq y \leq 3, \\
(x, y) \mid \begin{array}{c}
\text { and } y \in[0,1] \cup[2,3] \text { if } x \in[2,4], \\
\text { and } x \in[0,2] \cup[4,6] \text { if } y \in[1,2]
\end{array}
\end{array}\right\} .
$$

Assume the desired trajectory is a straight line described by

$$
p_{d}(t)=\left[\begin{array}{ll}
0.1 t, 0.05 t
\end{array}\right]^{T} .
$$

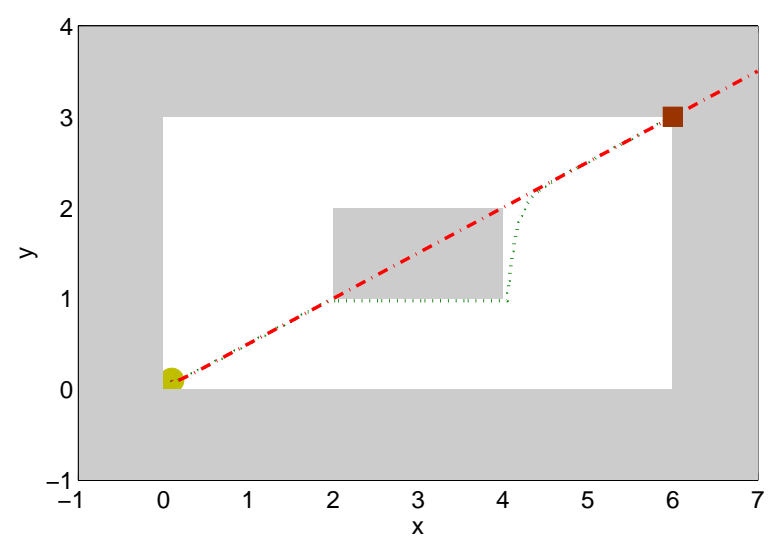

Fig. 4. $(x, y)$ trajectories of the system (1) for the set $\mathcal{P}_{a}$ given in (16): h-closed-loop (red, dashed-and-dotted) and s-closed-loop (green, dashed). Round mark: the initial position of the robot. Square mark: the final position of the robot with the shared-control.

Figure 4 shows that the $(x, y)$ trajectory of the system without shared-control goes through the non-admissible region (the grey shaded area in the center), while the $(x, y)$ trajectory of the s-closed-loop system moves around it and goes along the boundary of $\mathcal{P}_{a}$ until the configuration of the h-closed-loop system enters $\mathcal{P}_{a}$ again. After 60 s, the $(x, y)$ trajectory of the h-closed-loop leaves $\mathcal{P}_{a}$ as the red, dashedand-dotted, line in Figure 4 indicates, while the robot with the shared-control stops at the corner (the boundary) of the admissible region.

\section{CONCLUSIONS}

We have developed a solution to the shared-control problem for the kinematic model of a mobile robot. A hysteresisbased switch is used to unite the human input and the feedback control input based on the definitions of the sets $\mathcal{R}_{s}\left(v_{h}\right), \mathcal{R}_{h}\left(v_{h}\right)$ and $\mathcal{R}_{d}\left(v_{h}\right)$. Even though the sharedcontrol theory is designed for convex admissible configuration sets, it can also be applied to non-convex sets, as illustrated in Section IV-B. Two simple examples are given in Section IV to show the effectiveness of the shared-control. 

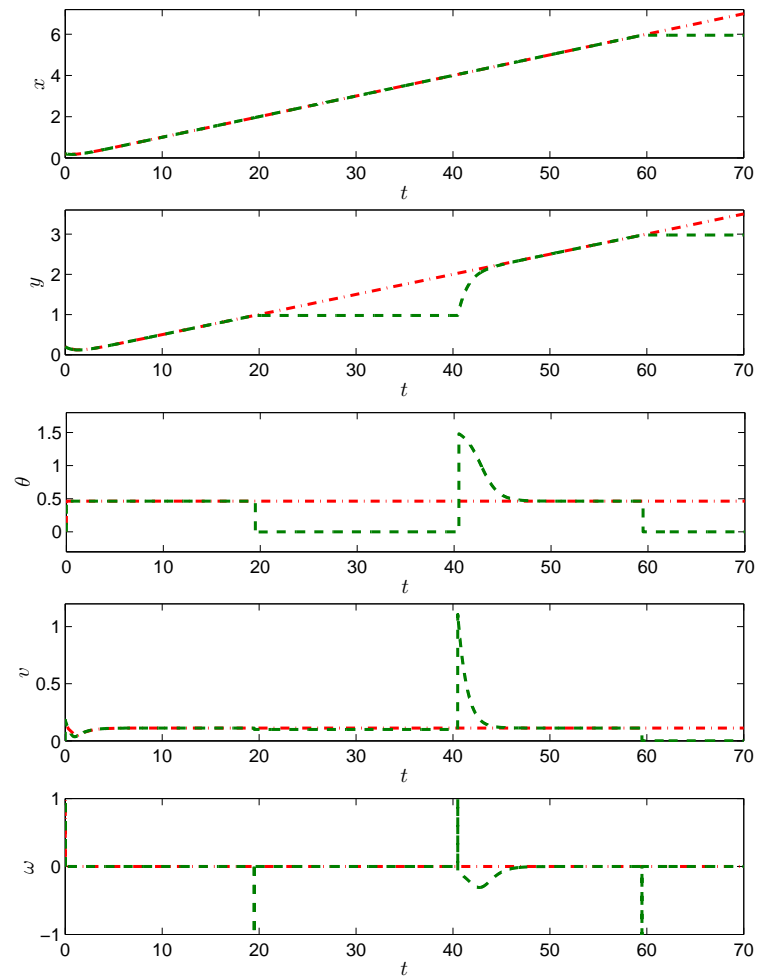

Fig. 5. Time histories of the variable $x, y, \theta, v$ and $\omega$ for the system (1) with the set $\mathcal{P}_{a}$ given by (16): h-closed-loop system (red, dashed-anddotted) and s-closed-loop system (green, dashed).

Future research will focus on four-wheel car-like systems and trailer systems.

\section{REFERENCES}

[1] P. Giguere and G. Dudek, "A simple tactile probe for surface identification by mobile robots," IEEE Trans. on Robotics, vol. 27, no. 3, pp. 534-544, 2011.

[2] T. Nguyen, N. N.P., and P. Tuong, "A study of pipe-cleaning and inspection robot," in Proc. of IEEE Conference on Robotics and Biomimetics, 2011, pp. 2593-2598.

[3] D. Voth, "A new generation of military robots," IEEE Trans. on Intelligent Systems, vol. 19, no. 4, pp. 2-3, 2004.

[4] R. Bogue, "Robots in the laboratory: a review of applications," Industrial Robot, vol. 39, no. 2, pp. 113-119, 2012.

[5] H. Chen, T. Fuhlbrigge, and X. Li, "Automated industrial robot path planning for spray painting process: A review," in Proc. of IEEE Conference on Automation Science and Engineering, 2008, pp. 522527.

[6] M. Pardowitz and R. Dillmann, "Towards life-long learning in household robots: The piagetian approach," in Proc. of IEEE Conference on Development and Learning, 2007, pp. 88-93.

[7] S. Parikh, V. Grassi, V. Kumar, and J. Okamoto, "Integrating human inputs with autonomous behaviors on an intelligent wheelchair platform," IEEE Intelligent Systems, vol. 22, no. 2, pp. 33-41, 2007.

[8] H. Boessenkool, D. Abbink, C. Heemskerk, F. Helm, and J. Wildenbeest, "A task-specific analysis of the benefit of haptic shared control during telemanipulation," IEEE Trans. on Haptics, vol. 6, no. 1, pp. $2-12,2013$.

[9] L. Saleh, P. Chevrel, F. Claveau, J. Lafay, and F. Mars, "Shared steering control between a driver and an automation: Stability in the presence of driver behavior uncertainty," IEEE Trans. on Intelligent Transportation Systems, vol. 14, no. 2, pp. 974-983, 2013.

[10] C. Cipriani, F. Zaccone, S. Micera, and M. Carrozza, "On the shared control of an EMG-controlled prosthetic hand: Analysis of userprosthesis interaction," IEEE Trans. on Robotics, vol. 24, no. 1, pp. 170-184, 2008.

[11] D. Bruemmer, D. Few, R. Boring, J. Marble, M. Walton, and C. Nielsen, "Shared understanding for collaborative control," IEEE Trans. on Systems, Man and Cybernetics, Part A: Systems and Humans, vol. 35, no. 4, pp. 494-504, 2005.

[12] B. McCarragher, G. Hovland, P. Sikka, and P. Aigner, "Hybrid dynamic modeling and control of constrained manipulation systems," IEEE Robotics \& Automation Magazine, vol. 4, no. 2, pp. 27-44, 1997.

[13] S. Nudehi, R. Mukherjee, and M. Ghodoussi, "A shared-control approach to haptic interface design for minimally invasive telesurgica training," IEEE Trans. on Control Systems Technology, vol. 13, no. 4, pp. 588-592, 2005.

[14] B. Khademian and K. Hashtrudi-Zaad, "Dual-user teleoperation systems: New multilateral shared control architecture and kinesthetic performance measures," IEEE Trans. on Mechatronics, vol. 17, no. 5, pp. 895-906, 2012.

[15] H. Kim, S. Biggs, D. Schloerb, J. Carmena, M. Lebedev, M. Nicolelis, and M. Srinivasan, "Continuous shared control for stabilizing reaching and grasping with brain-machine interfaces," IEEE Trans. on Biomedical Engineering, vol. 53, no. 6, pp. 1164-1173, 2006.

[16] A. Poncela, C. Urdiales, E. J. Perez, and F. Sandoval, "A new efficiency-weighted strategy for continuous human/robot cooperation in navigation," IEEE Trans. on Systems, Man and Cybernetics, vol. 39, no. 3, pp. 486-500, 2009.

[17] J. Jiang and A. Astolfi, "Shared-control for fully actuated linear mechanical systems," in Proc. of IEEE Conference on Decision and Control, Florence, Italy, 2013.

[18] — , "Output-feedback shared-control for fully actuated linear mechanical systems," in Proc. of American Control Conference, Portland, 2014.

[19] L. Zaccarian, "On dynamic control allocation for input-redundant control systems," in Proc. of IEEE Conference on Decision and Control, 2007, pp. 1192-1197.

[20] C. Prieur and A. Teel, "Uniting local and global output feedback controller," IEEE Trans. Automatic Control, vol. 56, no. 7, pp. 16361679,2011

[21] Y. Koren and J. Borenstein, "Potential field methods and their inherent limitations for mobile robot navigation," in Proc. of IEEE Conference on Robotics and Automation, 1991, pp. 1398-1404.

[22] K. Tee, S. Ge, and E. Tay, "Barrier Lyapunov functions for the control of output-constrained nonlinear systems," Automatica, vol. 45, pp. 918-927, 2009.

[23] C. Prieur, "Uniting local and global controllers with robustness to vanishing noise," Math. Control Signals Systems, vol. 14, pp. 143$172,2001$. 